\title{
The application of magnetic resonance imaging and diffusion-weighted imaging in the diagnosis of hypoxic-ischemic encephalopathy and kernicterus in premature infants
}

\author{
Lin Zhang ${ }^{1}$, Jin $\mathrm{Gao}^{2}$, Yibin Zhao ${ }^{3}$, Qian Zhang ${ }^{4}$, Jiantian Lu ${ }^{1}$, Xiaoyun Yang ${ }^{5}$ \\ ${ }^{1}$ Department of Radiology, Children's Hospital Affiliated to Kunming Medical University, Kunming, China; ${ }^{2}$ Department of Pediatrics, Children's \\ Hospital Affiliated to Kunming Medical University, Kunming, China; ${ }^{3}$ Rehabilitation Department, Children's Hospital Affiliated to Kunming \\ Medical University, Kunming, China; ${ }^{4}$ Clinical Laboratory, Children's Hospital Affiliated to Kunming Medical University, Kunming, China; \\ ${ }^{5}$ Neonatal Intensive Care Unit, Yuxi Children's Hospital, Yuxi, China \\ Contributions: (I) Conception and design: All authors; (II) Administrative support: All authors; (III) Provision of study materials or patients: All \\ authors; (IV) Collection and assembly of data: All authors; (V) Data analysis and interpretation: All authors; (VI) Manuscript writing: All authors; (VII) \\ Final approval of manuscript: All authors. \\ Correspondence to: Xiaoyun Yang. Neonatal Intensive Care Unit, Yuxi Children's Hospital, No. 19 Guishan Road, Hongta District, Yuxi 653100, \\ China. Email: yxyun0909@163.com.
}

Background: To explore the application of magnetic resonance imaging (MRI) and diffusion-weighted imaging (DWI) in the diagnosis of hypoxic-ischemic encephalopathy and kernicterus in premature infants.

Methods: Fifty-eight premature infants with hypoxic-ischemic encephalopathy and fifty-eight premature infants with kernicterus who were examined and treated in our hospital between January 2018 and January 2021 were assigned to the observation group or the control group. All patients were examined by MRI imaging and DWI imaging, and the examination results were compared between the two groups.

Results: No significant differences were found in sensitivity, specificity, positive predictive value, or negative predictive value between the observation group and the control group $(\mathrm{P}>0.05)$. MRI could clearly visualize the signal changes of patients, whereas DWI did not show any signal changes. There was no significant difference between MRI and DWI in the diagnosis of hypoxic-ischemic encephalopathy in premature infants. Further, there was no significant difference in the diagnostic performance of MRI between the observation group and the control group $(\mathrm{P}>0.05)$. However, the diagnostic performance of DWI in the control group was better than that in the observation group, and the difference was statistically significant $(\mathrm{P}<0.05)$.

Conclusions: MRI and DWI imaging have high detection rates for the diagnosis of hypoxic-ischemic encephalopathy and kernicterus in premature infants. These imaging methods can benefit the treatment of premature infants and have important clinical application value.

Keywords: Magnetic resonance imaging (MRI); diffusion-weighted imaging (DWI); hypoxic-ischemic encephalopathy in premature infants; kernicterus; diagnosis

Submitted Feb 23, 2021. Accepted for publication Apr 20, 2021.

doi: $10.21037 / \mathrm{tp}-21-128$

View this article at: http://dx.doi.org/10.21037/tp-21-128

\section{Introduction}

Kernicterus, also termed bilirubin encephalopathy, is a serious central nervous system condition that affects infants in the neonatal period. This condition, which is mainly caused by severe hyperbilirubinemia (1), can easily and severely affect the globus pallidus, ventral brainstem nuclei, and nerve nuclei of patients. A failure to deliver timely diagnosis and treatment leads to the aggravation 
of the patient's condition and death (2). Furthermore, for patients with kernicterus, there are inevitable sequelae (3). Neonatal hypoxic-ischemic encephalopathy, a persistent brain injury caused by neonatal asphyxia, can affect patients' neurological function $(3,4)$. Therefore, early diagnosis and treatment of hypoxic-ischemic encephalopathy is of critical clinical significance, and can effectively reduce the disability and mortality rates among patients (5).

Magnetic resonance imaging (MRI) has a high resolution to enable the diagnosis of intracranial lesions in children $(6,7)$. However, few studies have investigated the clinical application of MRI and diffusion-weighted imaging (DWI) in the diagnosis of Hypoxic-ischemic encephalopathy and kernicterus. To promote the progress of clinical diagnostic medicine, we conducted a diagnostic study of premature infants with hypoxic-ischemic encephalopathy and kernicterus who were examined and treated in our hospital in the period from January 2018 to January 2021. We present the following article in accordance with the STARD reporting checklist (available at http://dx.doi.org/10.21037/ tp-21-128).

\section{Methods}

\section{General patient information}

Fifty-eight premature infants with hypoxic-ischemic encephalopathy and 58 premature infants with kernicterus who were examined and treated in our hospital between January 2018 and January 2021 were assigned to the observation group or the control group. In the control group, there were 27 and 31 females and males, respectively. For these patients, the gestational age ranged from $27-40$ weeks, with an average gestational age of $33.5 \pm 6.5$ years, and their body weights ranged from $1.5-4.1 \mathrm{~kg}$, with an average body weight of $2.80 \pm 1.30 \mathrm{~kg}$. In the observation group, there were 29 females and 29 males. For these patients, the gestational age ranged from 26-40 weeks, with an average gestational age of $33 \pm 7$ years, and their body weights ranged from $1.5-4.2 \mathrm{~kg}$, with an average weight of $2.85 \pm 1.25 \mathrm{~kg}$. Comparison of the basic data of the control and observation patients revealed no differences, meaning the two groups were comparable $(\mathrm{P}>0.05)$. All procedures performed in this study involving human participants were in accordance with the Declaration of Helsinki (as revised in 2013). The study was approved by ethics board of Children's Hospital Affiliated to Kunming Medical University (NO.: IRB-2018011) and informed consent was taken from all the patients.
The inclusion criteria for patients were as follows: (I) a clinical diagnosis of hypoxic-ischemic encephalopathy or kernicterus of premature infants; (II) complete clinical data; and (III) the families of the patients were fully informed of the study and agreed to participate voluntarily. The exclusion criteria were: (I) accompanied with congenital heart disease; (II) unable or refused to cooperate with the research; and (III) patients who were due to receive surgery in the near future.

\section{MRI examination procedures}

All patients underwent MRI examination using an AVANTOI-CLASS $1.5 \mathrm{~T}$ superconducting MR scanner. The head was examined with a phased array coil, and chloral hydrate $(50 \mathrm{~mL} / \mathrm{kg})$ was used for enema before the examination. The patient was placed in the appropriate position, usually the supine position, with their ears plugged with cotton and their head fixed. It is worth noting that warming measures needed to be applied during the examination. The patients were scanned by cranial MRI. The scanning parameters were: (TR 2,000 ms, TE $9 \mathrm{~ms}$ ), slice thickness $4 \mathrm{~mm}$, layer spacing $1 \mathrm{~mm}$, and matrix $512 \times 512$.

All patients were examined by DWI with a single-shot spin echo planar imaging (SE-EPI) DWI sequence. The scanning parameters were (TR 5,400 ms, TE $75 \mathrm{~ms}$ ), and 0, 500 and $1,000 \mathrm{~s} / \mathrm{mm}^{2}$ were selected for scanning.

\section{Observation index}

After the imaging examination of patients in the observation and control groups, the MRI and DWI images were analyzed by experienced diagnostic physicians, and the results of the two groups were compared. The sensitivity, specificity, positive predictive value, and negative predictive value were determined, and the diagnostic coincidence rate was calculated.

\section{Statistical method}

Data were analyzed by SPSS 21.0 software (SPSS, Inc., Chicago, IL,USA). Measurement data were compared by Student's $t$-test and expressed as mean \pm standard deviation $(\bar{x} \pm s)$. Count data were analyzed using the $\chi^{2}$ test, and expressed by rate (\%). $\mathrm{P}<0.05$ was considered to represent a significant difference. 
Table 1 Comparison of MRI and DWI results between the two groups ( $\mathrm{n}=58$ )

\begin{tabular}{lcccc}
\hline Indexes & Observation group & Control group & $\chi^{2}$ & $P$ \\
\hline Sensitivity (\%) & 49 & 50 & 0.069 & 0.793 \\
Specificity (\%) & 48 & 47 & 0.058 & 0.809 \\
Positive predictive value (\%) & 51 & 50 & 0.077 & 0.782 \\
Negative predictive value (\%) & 53 & 50 & 0.78 & 0.377 \\
\hline
\end{tabular}

$\mathrm{MRI}$, magnetic resonance imaging; DWI, diffusion-weighted imaging.
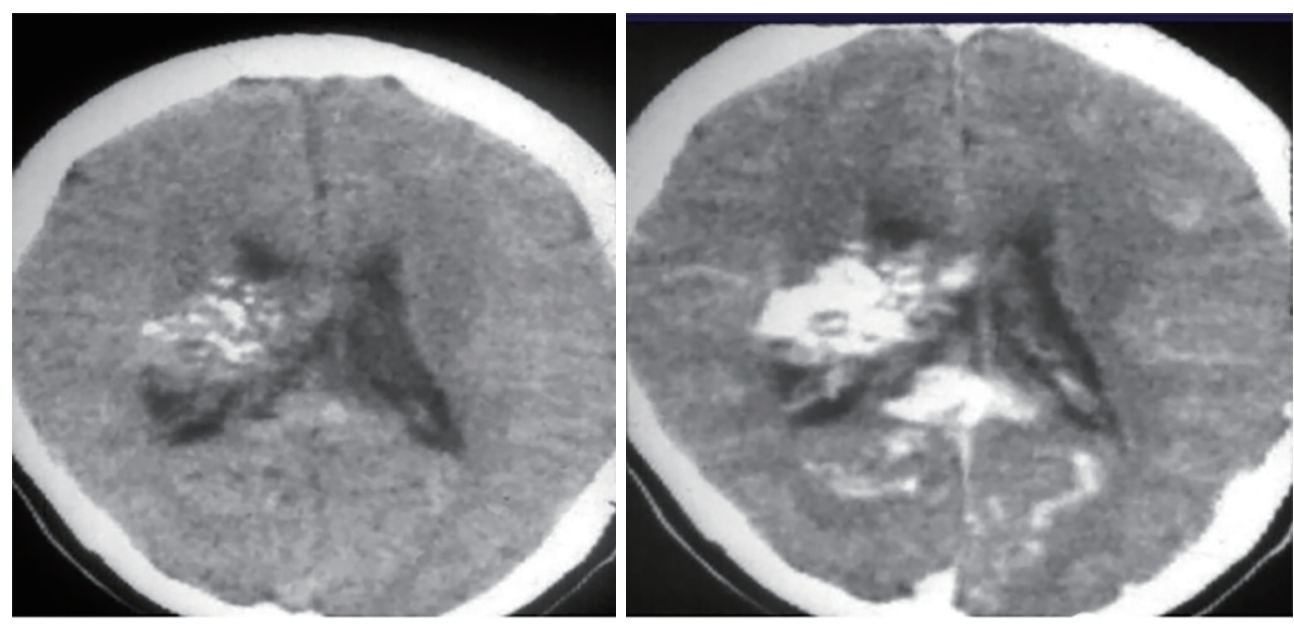

Figure 1 Imaging results of kernicterus in a premature infant.

\section{Results}

Comparison of the results of MRI and DWI between the two groups

As shown in Table 1, no significant difference was found in sensitivity, specificity, positive predictive value, or negative predictive value between the observation and control groups $(\mathrm{P}>0.05)$. Representative images of cases with kernicterus and hypoxic-ischemic encephalopathy are shown in Figures 1 and 2, respectively. For patients with kernicterus in Figure 1, DWI detected no abnormalities; however, MRI revealed the bilateral globus pallidus to be significantly increased, with a blurred boundary. In the patients with hypoxicischemic encephalopathy in Figure 2, the main observations were diffuse edema, low signal intensity in the white matter around the occipital lobe, and narrowing of the sulcus and ventricle.

\section{The globus pallidus in different groups}

Table 2 shows a comparison of MRI and DWI in depicting

the condition of the globus pallidus in the patients. MRI could clearly visualize the signal changes in patients, whereas DWI did not show any signal changes.

\section{Comparison of MRI and DWI results of patients in the observation group}

As shown in Table 3, no significant difference existed between MRI and DWI in the diagnosis of hypoxicischemic encephalopathy in premature infants in the observation group $(\mathrm{P}>0.05)$.

\section{The diagnostic performance of MRI}

As shown in Table 4, the diagnostic performance of MRI did not differ significantly between the observation group and the control group $(\mathrm{P}>0.05)$.

\section{The diagnostic performance of DWI}

As shown in Table 5, the diagnostic performance of DWI 


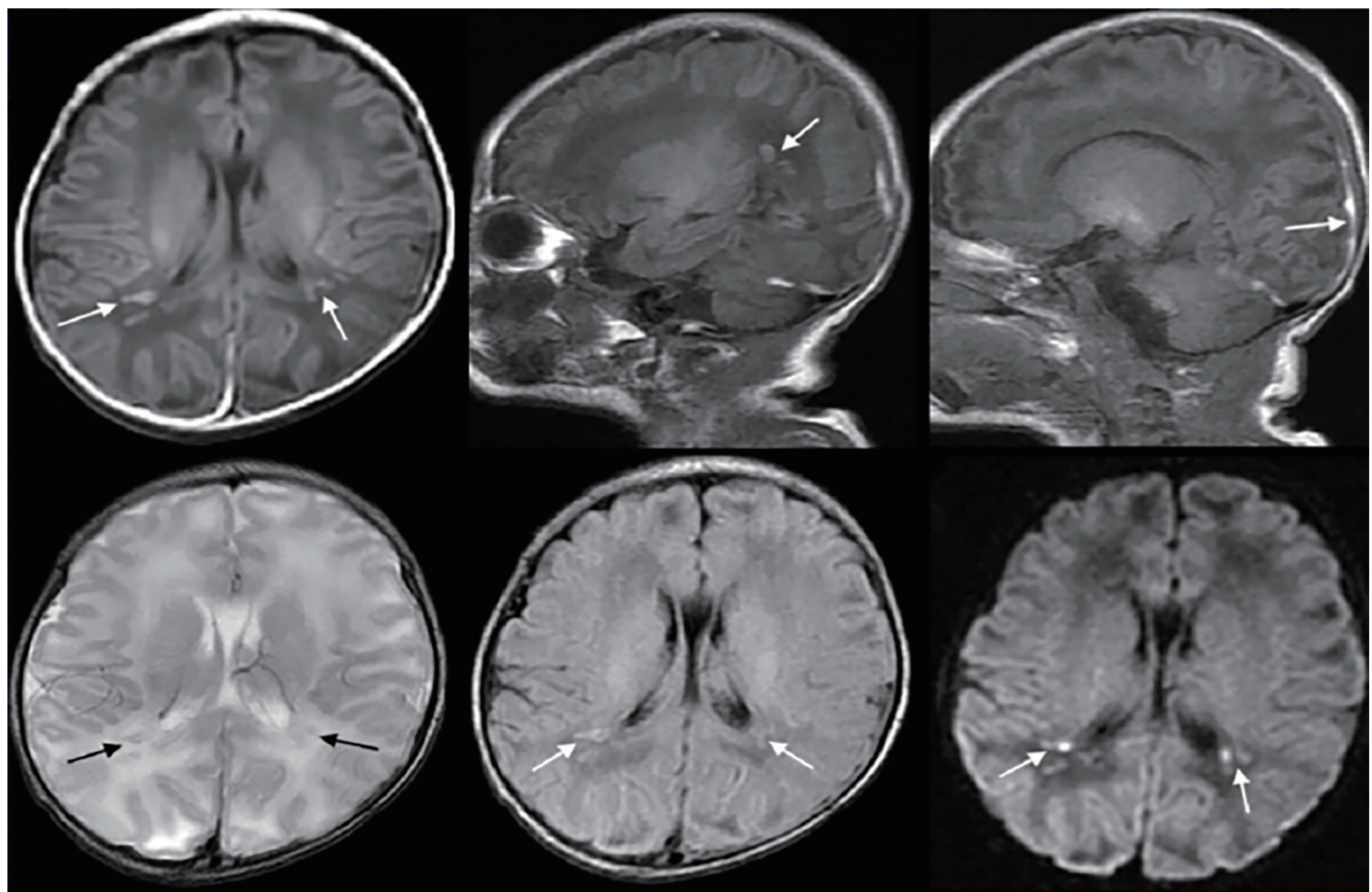

Figure 2 Imaging results of hypoxic-ischemic encephalopathy in a premature infant. The arrows indicate location of ischemic hypoxic encephalopathy in premature infants.

Table 2 The condition of globus pallidus in the control group $(\mathrm{n}=58)$

\begin{tabular}{lcccc}
\hline Indexes & MRI & DWI & $\chi^{2}$ & P \\
\hline Signal change & 27 & 5 & 20.887 & 0.000 \\
Significantly increased & 19 & 7 & 7.139 & 0.008 \\
No significant increase & 11 & 6 & 1.723 & 0.189 \\
No change & 1 & 40 & 57.378 & 0.000 \\
\hline
\end{tabular}

was better in the control group than in the observation group, and the difference was statistically significant $(\mathrm{P}<0.05)$.

\section{Discussion}

Neonatal hypoxic-ischemic encephalopathy is a serious complication in clinical medicine. Although its pathogenesis has not been fully explained, relevant research results indicate that it is largely related to placental abnormalities, heart failure and other factors (8-10). When neonatal hypoxic-ischemic encephalopathy occurs, the patient experiences gas exchange disturbance. In the neonatal period, the brain tissue is in the stage of growth and development, and the metabolic ability is strong. The occurrence of symptoms such as ischemia or hypoxia can cause lactic acid to accumulate in the brain tissue, resulting in brain atrophy (11). Cases of intracranial hemorrhage in patients with neonatal hypoxic-ischemic encephalopathy can be divided into intraventricular hemorrhage, subdural hemorrhage, cerebral parenchyma hemorrhage $(12,13)$. Therefore, to effectively avoid the occurrence of above symptom, it is essential that patients are diagnosed and treated early to reduce the occurrence of adverse complications, and promote better recovery (14).

Bilirubin is an important factor causing bilirubin 
Table 3 Comparison of the results of MRI and DWI in the observation group ( $\mathrm{n}=58)$

\begin{tabular}{lcccc}
\hline Indexes & MRI & DWI & $\chi^{2}$ & P \\
\hline Intracranial hemorrhage & 21 & 20 & 0.038 & 0.188 \\
Delayed myelination of white matter & 15 & 13 & 0.051 & 0.664 \\
Brain perforation malformation & 12 & 13 & 0.821 \\
Caudate nucleus abnormality & 10 & 12 & 0.224 & 0.636 \\
\hline
\end{tabular}

$\mathrm{MRI}$, magnetic resonance imaging; DWI, diffusion-weighted imaging.

Table 4 The diagnostic performance of MRI $(n=58)$

\begin{tabular}{lcccc}
\hline Indexes & Observation group & Control group & $\chi^{2}$ & $P$ \\
\hline High signal & 33 & 32 & 0.035 & 0.852 \\
Slightly higher signal & 21 & 18 & 0.348 & 0.555 \\
Equal signal & 4 & 8 & 1.487 & 0.223 \\
\hline
\end{tabular}

MRI, magnetic resonance imaging.

Table 5 The diagnostic performance of DWI $(n=58)$

\begin{tabular}{lcccc}
\hline Indexes & Observation group & Control group & $\chi^{2}$ & $P$ \\
Visible edema area & 27 & 39 & 5.062 & 0.024 \\
Invisible & 31 & 19 & 5.062 & 0.024 \\
\hline
\end{tabular}

DWI, diffusion-weighted imaging.

encephalopathy. At present, there are three kinds of bilirubin monitoring in children with hyperbilirubinemia: venous blood biochemical analysis, trace blood bilirubin determination and percutaneous bilirubin determination. Percutaneous bilirubin monitoring is non-invasive and easy to operate. At present, it is mainly used for early screening, monitoring and phototherapy evaluation of children with jaundice. There is no significant difference between the determination of trace blood bilirubin and the value of bilirubin measured by venous blood biochemical analysis. The result of micromethod is accurate, easy to operate, easy to operate and less damage. It is not affected by the severity of bilirubin and skin factors, which is suitable for close monitoring of the level of bilirubin in blood. Continuous monitoring of blood S100B and Tau protein levels can guide the treatment of children with hyperbilirubinemia and further prevent the occurrence of bilirubin encephalopathy. Creatine kinase brain isozyme is one of the sensitive and objective indexes for early diagnosis of bilirubin brain injury. Magnetic resonance imaging is one of the more mature methods for the diagnosis of bilirubin encephalopathy at present, and most of the diagnosis of acute and chronic bilirubin encephalopathy can be determined by MRI. Magnetic resonance spectroscopy is more sensitive than conventional MRI, so it can be used for early detection of brain injury caused by bilirubin neurotoxicity. At present, it is advocated that routine MRI and magnetic resonance spectroscopy should be combined to monitor the damage of nerve tissue in time and comprehensively, so as to better guide clinical intervention and rehabilitation prognosis. The abnormal change of brainstem auditory evoked potential is one of the common manifestations of bilirubin encephalopathy, and it can also be the only manifestation of bilirubin-induced neurological dysfunction, and it is one of the earliest and lightest manifestations of bilirubin neurotoxicity. Brainstem and VIII brain nerves are particularly sensitive to bilirubin toxicity III. Neonatal neurobehavioral score can be used for early diagnosis of neonatal bilirubin encephalopathy, and its sensitivity to hearing impairment is even better than that of auditory brainstem response. The advantages of neonatal neurobehavioral score are: sensitive, reliable, repeated 
testing will not cause damage to newborns, easy to operate, no need for special technical training, the tools required are simple and economical, and are easy to be widely used in clinic. EEG can detect abnormal brain function as early as possible through the real-time recording of EEG activity, so it can be used in one of the early diagnosis of brain injury caused by high bilirubin, especially the application of amplitude integrated EEG. It provides a new means for neonatal nerve development and brain injury, in order to guide early clinical intervention and disease prognosis evaluation.

Neonatal kernicterus mainly occurs due to serious complications caused by hyperbilirubinemia in the neonatal period and has an immense adverse impact on the lives of infants. If positive and effective treatment measures are not taken, the risk of sequelae increases, and severe cases can even prove life-threatening $(15,16)$. The clinical symptoms of kernicterus are usually yellowing of the skin, lethargy, and limb convulsions (17); however, due to the particularity of the newborn, it shows different physiological jaundice in the neonatal period, which is difficult to distinguish to a large extent. Therefore, misdiagnosis can easily happen, which is not conducive to the timely treatment of patients, which requires an early clinical diagnosis (18). Pathological injury in the neonatal period can lead to the accumulation of a large number of nuclei in the central nervous system, including the globus pallidus, which has the most vulnerable nuclei. In patients with abnormal bilirubin, the signal intensity of the globus pallidus will show an increasing trend $(19,20)$. Therefore, positive and effective diagnostic imaging can aid in the accurate assessment of the condition and form a basis for clinical diagnosis, which facilitates timely treatment, thus avoiding serious damage to the patient's health.

As a tomographic imaging technology, MRI has the characteristics of high resolution and imaging diversity for clinical diagnosis, and its clinical application does not expose the patient to unnecessary radiation damage (21). MRI can clearly show the focus range of the patient, and provide a diagnostic basis for clinical diagnosis and treatment (22). 3.0TMR high resolution imaging technology combines multi-channel body coils and respiratory instrumentation techniques to help reduce the production of motion artifacts in breathing, improve image clarity. 3.0TMR high resolution imaging is applied to nuclear jaundice and hypoxic ischemic encephalopathy. It is accurately judging the severity of the condition, which is conducive to the improvement of the development and prognosis effect of the treatment plan. Patients with hypoxic-ischemic encephalopathy have a high probability of brain edema, which is mainly caused by the continuous increase of brain water content after the occurrence of asphyxia or other conditions. As an early symptom, this kind of complication may affect the surrounding tissue and result in sequelae, even affecting patients' life and health $(23,24)$. In clinical practice, routine computed tomography (CT) has a very limited value for diagnosing neonatal kernicterus; furthermore, it involves some radiation exposure. Using MRI technology to diagnose patients can not only effectively reduce the radiation damage to patients, but it can also accurately highlight abnormal signals of the globus pallidus in patients. It is worth noting that great similarity exists between signals and the globus pallidus during neonatal development. Lack of a certain discrimination point is also the limitation of MRI in clinical application $(25,26)$. Therefore, the use of this method alone cannot effectively achieve a scientific diagnosis; thus, it needs to be combined with other methods to improve the diagnostic accuracy, and DWI technology is used more frequently in clinic (27). In terms of DWI technology, conventional CT and other examination methods can be used to diagnose and evaluate brain injury; however, conventional CT cannot detect mild brain edema in time, and conventional CT lacks accuracy and sensitivity, so its use is extremely limited. Therefore, brain injury needs to be diagnosed and monitored more accurately in a more reasonable way $(25,28,29)$. DWI technology has a certain value in the diagnosis of neonatal brain tissue damage. However, it is worth mentioning that due to the high water content in the brain tissue of newborns, it is often impossible to accurately evaluate the scope and degree of brain injury when ischemia has led to the dispersion of water molecules in patients (30). In the face its limitations, it is necessary to enhance the diagnostic accuracy of this imaging method through its joint application with MRI technology. Therefore, in the diagnostic examination of premature infants with hypoxicischemic encephalopathy and kernicterus, when used alone, MRI or DWI technology cannot accurately depict the brain tissue. However, by effectively combining the two methods, we can improve the scientific basis of diagnosis, provide scientific guidance to inform early treatment, and reduce the occurrence of misdiagnosis. Furthermore, the joint application of the two methods can effectively improve the quality of treatment and the overall reputation of the hospital.

Studies have shown that MRI examination has positive 
significance for assessing the nerves in brain tissue. MRI generates images of the internal organs of the human body using a magnetic field and radio waves. Due to its good efficacy, it has a wide range of clinical applications (31). DWI technology also has a clear positive significance for clinical diagnosis, and can be used to effectively assess clinical symptoms, such as brain edema, and provide a scientific basis for clinical treatment (32). In this study, 58 premature infants with hypoxic-ischemic encephalopathy and 58 premature infants with kernicterus were selected for evaluation using these two diagnostic methods, and their imaging results were compared. There was no significant difference in sensitivity, specificity, positive predictive value, or negative predictive value between the observation group and the control group $(\mathrm{P}>0.05)$. Therefore, on the whole, in the diagnosis and treatment of premature infants with hypoxic-ischemic encephalopathy or kernicterus, we need to take positive and effective approaches to obtain an early diagnosis, and the combined use of MRI and DWI can effectively improve the diagnostic accuracy for such patients (33). However, it is worth noting that, although this approach can effectively improve the diagnostic efficiency, the pathological features and the degree of pathological change in patients can vary greatly due to the differences between individuals and their clinical symptoms (34-36). Furthermore, after the occurrence of hypoxicischemic encephalopathy, patients are prone to intracranial hemorrhage and brain edema. In clinical diagnosis, the degree of brain tissue injury cannot be evaluated in detail, and patients need to be clinically assessed before diagnosis $(37,38)$. Furthermore, treatment and other intervention factors can affect the accuracy of MRI $(39,40)$. Therefore, in clinical diagnosis, it is necessary to make a sound assessment based on the patient's condition in order to reduce the risk of misdiagnosis and the effect of other factors which are not conducive to the timely treatment of the patient.

In conclusion, MRI and DWI imaging have a high detection rate for the diagnosis of hypoxic-ischemic encephalopathy and kernicterus in premature infants. The combined application of these two methods can make up for their limitations individually, improve the diagnostic accuracy, and provide a scientific basis for diagnosis, which is conducive to the treatment of patients and can better promote their recovery. The combined use of MRI and DWI has positive significance for improving the overall quality of treatment and can benefit the doctor-patient relationship; therefore, this method has important clinical application value.

\section{Acknowledgments}

Funding: This project is a joint special fund project of Yunnan Provincial Department of science and technology and Kunming Medical University [2018FE001(-099)].

\section{Footnote}

Reporting Checklist: The authors have completed the STARD reporting checklist. Available at http://dx.doi.org/10.21037/ tp-21-128

Data Sharing Statement: Available at http://dx.doi. org/10.21037/tp-21-128

Conflicts of Interest: All authors have completed the ICMJE uniform disclosure form (available at http://dx.doi. org/10.21037/tp-21-128). The authors have no conflicts of interest to declare.

Ethical Statement: The authors are accountable for all aspects of the work in ensuring that questions related to the accuracy or integrity of any part of the work are appropriately investigated and resolved. All procedures performed in this study involving human participants were in accordance with the Declaration of Helsinki (as revised in 2013). The study was approved by ethics board of Children's Hospital Affiliated to Kunming Medical University (NO.: IRB-2018-011) and informed consent was taken from all the patients.

Open Access Statement: This is an Open Access article distributed in accordance with the Creative Commons Attribution-NonCommercial-NoDerivs 4.0 International License (CC BY-NC-ND 4.0), which permits the noncommercial replication and distribution of the article with the strict proviso that no changes or edits are made and the original work is properly cited (including links to both the formal publication through the relevant DOI and the license). See: https://creativecommons.org/licenses/by-nc-nd/4.0/.

\section{References}

1. Variane GFT, Magalhães M, Gasperine R, et al. Early amplitude-integrated electroencephalography for monitoring neonates at high risk for brain injury. J Pediatr (Rio J) 2017;93:460-6.

2. Pisani F, Spagnoli C. Monitoring of newborns at high risk 
for brain injury. Ital J Pediatr 2016;42:48.

3. Zaghloul N, Kurepa D, Bader MY, et al. Prophylactic inhibition of NF-kappaB expression in microglia leads to attenuation of hypoxic ischemic injury of the immature brain. J Neuroinflammation 2020;17:365.

4. Novak CM, Ozen M, Burd I. Perinatal Brain Injury: Mechanisms, Prevention, and Outcomes. Clin Perinatol 2018;45:357-75.

5. Dorner R A, Burton VJ, Allen MC, et al. Preterm neuroimaging and neurodevelopmental outcome: a focus on intraventricular hemorrhage, post-hemorrhagic hydrocephalus, and associated brain injury. J Perinatol 2018;38:1431-43.

6. Wisnowski JL, Panigrahy A, Painter MJ, et al. Magnetic Resonance Imaging Abnormalities in Advanced Acute Bilirubin Encephalopathy Highlight Dentato-ThalamoCortical Pathways. J Pediatr 2016;174:260-3.

7. Back SA. White matter injury in the preterm infant: pathology and mechanisms. Acta Neuropathol 2017;134:331-49.

8. Rao R, Trivedi S, Vesoulis Z, et al. Safety and ShortTerm Outcomes of Therapeutic Hypothermia in Preterm Neonates 34-35 Weeks Gestational Age with HypoxicIschemic Encephalopathy. J Pediatr 2017;183:37-42.

9. Kale A, Joshi P, Kelkar AB. Restricted diffusion in the corpus callosum: A neuroradiological marker in hypoxicischemic encephalopathy. Indian J Radiol Imaging 2016;26:487-92.

10. Karimzadeh P, Fallahi M, Kazemian M, et al. Bilirubin Induced Encephalopathy. Iran J Child Neurol 2020;14:7-19.

11. Donneborg ML, Hansen BM, Vandborg PK, et al. Extreme neonatal hyperbilirubinemia and kernicterus spectrum disorder in Denmark during the years 20002015. J Perinatol 2020;40:194-202.

12. Yang J, Wu EX. Detection of cortical gray matter lesion in the late phase of mild hypoxic-ischemic injury by manganese-enhanced MRI. Neuroimage 2008;39:669-79.

13. Matsufuji M, Sano N, Tsuru H, et al. Neuroimaging and neuropathological characteristics of cerebellar injury in extremely low birth weight infants. Brain Dev 2017;39:735-42.

14. Choi J Y, Choi YS, Rha DW, et al. The clinical outcomes of deep gray matter injury in children with cerebral palsy in relation with brain magnetic resonance imaging. Res Dev Disabil 2016;55:218-25.

15. van Toorn R, Brink P, Smith J, et al. Bilirubin-Induced Neurological Dysfunction: A Clinico-RadiologicalNeurophysiological Correlation in 30 Consecutive
Children. J Child Neurol 2016;31:1579-83.

16. Alam A, Sahu S. Magnetic Resonance Imaging in Evaluation of Periventricular Leukomalacia. Med J Armed Forces India 2010;66:374-80.

17. Dağ Y, Firat AK, Karakas HM, et al. Clinical outcomes of neonatal hypoxic ischemic encephalopathy evaluated with diffusion-weighted magnetic resonance imaging. Diagn Interv Radiol 2006;12:109-14.

18. Chang LL, Wynn JL, Pacella MJ, et al. Enteral Feeding as an Adjunct to Hypothermia in Neonates with HypoxicIschemic Encephalopathy. Neonatology 2018;113:347-52.

19. Ali A, Yalcin R, Unluer-Gumustas A. Cranial MR characteristics of Cerebral Palsy cases and correlation of findings with clinical results. Turk J Pediatr 2019;61:525-37.

20. Ayrapetyan M, Talekar K, Schwabenbauer K, et al. Apgar Scores at 10 Minutes and Outcomes in Term and Late Preterm Neonates with Hypoxic-Ischemic Encephalopathy in the Cooling Era. Am J Perinatol 2019;36:545-54.

21. Chang MY, Borchert MS. Advances in the evaluation and management of cortical/cerebral visual impairment in children. Surv Ophthalmol 2020;65:708-24.

22. Tortora D, Mattei PA, Navarra R, et al. Prematurity and brain perfusion: Arterial spin labeling MRI. Neuroimage Clin 2017;15:401-7.

23. Winter JD, Thompson RT, Gelman N. Efficacy of motion artifact reduction in neonatal DW segmented EPI at $3 \mathrm{~T}$ using phase correction by numerical optimization and segment data swapping. Magn Reson Imaging 2007;25:1283-91.

24. Zhang F, Liu C, Qian L, et al. Diffusion Tensor Imaging of White Matter Injury Caused by Prematurity-Induced Hypoxic-Ischemic Brain Damage. Med Sci Monit 2016;22:2167-74.

25. Sewell EK, Andescavage NN. Neuroimaging for Neurodevelopmental Prognostication in High-Risk Neonates. Clin Perinatol 2018;45:421-37.

26. Spagnoli C, Falsaperla R, Deolmi M, et al. Symptomatic seizures in preterm newborns: a review on clinical features and prognosis. Ital J Pediatr 2018;44:115.

27. Kitai Y, Hirai S, Okuyama N, et al. Diagnosis of Bilirubin Encephalopathy in Preterm Infants with Dyskinetic Cerebral Palsy. Neonatology 2020;117:73-9.

28. Cece H, Abuhandan M, Cakmak A, et al. Diffusionweighted imaging of patients with neonatal bilirubin encephalopathy. Jpn J Radiol 2013;31:179-85.

29. Yuan X, Song J, Gao L, et al. Early Amplitude-Integrated Electroencephalography Predicts Long-Term Outcomes in Term and Near-Term Newborns With Severe 
Hyperbilirubinemia. Pediatr Neurol 2019;98:68-73.

30. Vitale V, Rossi E, Di Serafino M, et al. Pediatric encephalic ultrasonography: the essentials. J Ultrasound 2020;23:127-37.

31. Moss HG, Jenkins DD, Yazdani M, et al. Identifying the translational complexity of magnetic resonance spectroscopy in neonates and infants. NMR Biomed 2019;32:e4089.

32. Bozzao A, Di Paolo A, Mazzoleni C, et al. Diffusionweighted MR imaging in the early diagnosis of periventricular leukomalacia. Eur Radiol 2003;13:1571-6.

33. Wang $X, W u$ W, Hou BL, et al. Studying neonatal bilirubin encephalopathy with conventional MRI, MRS, and DWI. Neuroradiology 2008;50:885-93.

34. Laptook AR. Birth Asphyxia and Hypoxic-Ischemic Brain Injury in the Preterm Infant. Clin Perinatol 2016;43:529-45.

35. Assefa Neknek G, Woldemichael K, Moges A, et al. MRI of bilirubin encephalopathy (kernicterus): A case series of 4 patients from Sub-Saharan Africa, May 2017. Radiol Case
Rep 2018;13:676-9.

36. Prabhakar N, Ahuja CK, Khandelwal N. B/L Basal Ganglia Lesions in a Child Leading to a Diagnosis of Glucose-6-Phosphate Dehydrogenase Deficiency. Ann Neurosci 2018;25:50-2.

37. Ribeiro BN, Lima GA, Ventura N, et al. Chronic kernicterus: magnetic resonance imaging findings. Radiol Bras 2016;49:407-8.

38. Lemmon ME, Boss RD, Bonifacio SL, et al. Characterization of Death in Neonatal Encephalopathy in the Hypothermia Era. J Child Neurol 2017;32:360-5.

39. Liao WH, Wang XY, Wu WL, et al. Differentiation of hypoxic-ischemic encephalopathy and acute bilirubin encephalopathy with magnetic resonance imaging in neonates. Zhongguo Dang Dai Er Ke Za Zhi 2009;11:181-4.

40. Watchko JF. Bilirubin-Induced Neurotoxicity in the Preterm Neonate. Clin Perinatol 2016;43:297-311.

(English Language Editor: J. Reynolds)
Cite this article as: Zhang L, Gao J, Zhao Y, Zhang Q, Lu J, Yang $X$. The application of magnetic resonance imaging and diffusion-weighted imaging in the diagnosis of hypoxicischemic encephalopathy and kernicterus in premature infants. Transl Pediatr 2021;10(4):958-966. doi: 10.21037/tp-21-128 